\title{
Special issue on self-organised construction
}

\author{
Heiko Hamann ${ }^{1}$ (D) Sebastian von Mammen $^{2}$. \\ Ingo Mauser $^{3}$
}

Received: 8 December 2017 / Accepted: 20 December 2017 / Published online: 4 January 2018

(C) Springer Science+Business Media, LLC, part of Springer Nature 2018

This special issue of the Swarm Intelligence journal is a follow-up of the workshop on Self-Organising Construction, which was held in conjunction with the IEEE International Conference on Self-Adaptive and Self-Organizing Systems (SASO) in Augsburg, Germany, in September 2016. The workshop and this special issue bring a wide variety of methods and results from different fields together. They all aim at the same overarching goal: understanding, retracing and making self-organising construction processes technologically viable.

The foundational biological perspective on the topic unearths insights about processes, such as nest constructions by social insects. Computer scientists translate and augment the biological principles to coordinated algorithms of collaboration. Engineers aim at providing the hardware platforms for robotic units which are able to transport and place construction materials. And there is the architectural perspective that explores how self-organised construction can complement and innovate established approaches to design, construction and even works of art. At the bottom of all these efforts lies the conceptual paradigm shift towards local interaction and local information in distributed systems: large numbers of individuals contribute to the construction process not by following a predefined plan but by sensing and reacting to stimuli in their local environment. Only carefully coordinated local actions allow for emerging global structures that show global patterns and have appropriate functions. Recently, with the wake of robotic swarms, self-organised construction is quickly gaining significance in the context of various design and construction processes.

\eiko Hamann

hamann@iti.uni-luebeck.de

Sebastian von Mammen

sebastian.von.mammen@uni-wuerzburg.de

Ingo Mauser

mauser@kit.edu

1 Institute of Computer Engineering, University of Lübeck, Lübeck, Germany

2 Department of Computer Science, University of Würzburg, Würzburg, Germany

3 Institute of Applied Informatics and Formal Description Methods, Karlsruhe Institute of Technology, Karlsruhe, Germany 
We are convinced that providing a common platform for the promotion of scientific efforts in this field will foster the transition towards real-world applications of self-organised construction. Each article accepted for this special issue has undergone a rigorous peer-review process by at least three referees. The referees were chosen in accordance with their expertise and international standing. Great care was given to identify and win the best possible referees to evaluate the works.

For this special issue, we selected four articles with investigative objectives ranging from the realisation of indirect communication of agents and problems related to their heterogeneity over architectural design and construction of structures by self-organising robots to complexity analysis of automated controller design algorithms. We start from a biological perspective on construction, proceed with a study that has a theoretical bias, followed by a paper with engineering focus, to end with the application of self-organised construction to architecture.

In "Can individual heterogeneity influence self-organised patterns in the termite nest construction model?", Saffre et al. investigate the impact of individual characteristics of termites on the construction of their nests. Their basic research efforts build on preceding models of termite nest construction and yield results applicable to a wide range of selforganising systems, especially in the context of real-world applications. Their investigations based on simulation show that global effects in self-organising systems can be adjusted by the populations' degrees of heterogeneity. In their study, changing the response thresholds of individual termites consistently led to greater numbers of pillars built. Deeper analysis shows that the observed behaviour emerges from the interplay of asymmetrical interactions at the individual level. The authors draw the conclusion that the relationships between individual rules, individual thresholds and the emergent results in technical systems have to be closely analysed and designed with great caution.

In "Putting it together: the computational complexity of designing robot controllers and environments for distributed construction", Wareham and Vardy analyse the complexity of problems related to target structures and to the design and verification of robot controllers in their environments. For this purpose, they use a finite-state robot controller model moving robots deterministically in a grid-based environment. In general, these problems may not be solved efficiently. However, for verification and design problems the authors show that certain restrictions on the combination of parameters, such as the number of robots per team and the sensor range of robots, help to make them tractable. In so doing, the authors work on the issue of automated controller design algorithms that design dependable and useful robot controllers, which are able to create arbitrary target structures with a high probability.

In "Local force cues for strength and stability in a distributed robotic construction system", Melenbrink and Werfel focus on the issue of stability during building processes and present a scenario in which a system of climbing robots has to build a cantilever across a gap in a two-dimensional simulation, ultimately leading to the construction of a complex structure, such as a bridge. They show that access to certain local information about forces works as an indirect and thus stigmergic communication mechanism that is crucial to the anticipation and prevention of structural failures. In their work, they consider both structures that may topple and anchored structures, which are built by agents using three different algorithms. Although the proposed algorithms do not yet provide stable solutions in unstructured and irregular environments, the authors conclude that the algorithms help to improve decentralised construction by adapting to unknown and changing conditions.

In "An interactive agent-based framework for materialization-informed architectural design", Groenewolt et al. present a framework that aims at the integration of self-organised agent-based models with architectural design methods. In light of established architectural 
design processes, they explore the means to interactively define and analyse self-organising designs in the context of the innovative and widely used Rhinoceros modelling software.

As a use-case scenario, they define rules for individual polyhedron agents to organise their alignment to form an architectural surface. The designer can interactively change the agents' states while the simulation is running and thereby play a part in the emergent design process. To demonstrate an exemplary workflow of a comprehensive design project, the authors present and discuss the results of the generated designs in light of various architecturally important measures.

The four articles in this special issue exhibit a broad variety of topics and lay grounds for further research efforts in the field of self-organised construction, related to the design and realisation of any structure, building, or infrastructure up to the scale of entire cities. We thank the authors very much for their contributed papers, the reviewers for their precise and helpful comments, and the editor in chief of the Swarm Intelligence journal, who assisted us in the production of this special issue and provided valuable feedback.

December 2017

Heiko Hamann

Sebastian von Mammen

Ingo Mauser 